FORMATION Formation emploi

Revue française de sciences sociales

101 | janvier-mars 2008

Numéro anniversaire : Regards croisés sur les

relations formation-emploi

\title{
Justesse et justice dans les recrutements
}

Fairness and rightness in recruitment practices

Korrekte und gerechte Stellenbesetzung

Justeza y justicia en los reclutamientos

\section{François Eymard-Duvernay}

\section{(2) OpenEdition}

1 Journals

Édition électronique

URL : http://journals.openedition.org/formationemploi/1051

DOI : 10.4000/formationemploi.1051

ISSN : 2107-0946

Éditeur

La Documentation française

Édition imprimée

Date de publication : 1 janvier 2008

Pagination : $55-70$

ISSN : 0759-6340

\section{Référence électronique}

François Eymard-Duvernay, « Justesse et justice dans les recrutements », Formation emploi [En ligne], 101 | janvier-mars 2008, mis en ligne le 31 mars 2010, consulté le 30 octobre 2020. URL : http:// journals.openedition.org/formationemploi/1051; DOI : https://doi.org/10.4000/formationemploi.1051 


\title{
Numéro anniversaire
}

\section{Justesse et justice dans les recrutements ${ }^{1}$}

\author{
Par François Eymard-Duvernay*
}

\author{
Recruter implique de mesurer correctement des compétences. \\ Ce problème d'information bien connu est compliqué par deux autres dimensions \\ de l'évaluation : son inscription dans des contextes sociaux spécifiques \\ et sa référence à des principes de justice.
}

Qu'est-ce qu'un recrutement équitable? Mon propos se situe à l'intersection des questions d'efficience et de justice. La « justesse » de l'évaluation renvoie à sa dimension cognitive, soit l'exercice de la rationalité. Mais cette approche est incomplète : l'évaluation ne peut être isolée de situations de coordination, qui induisent des questions de justice. Il est habituel de distinguer artificiellement deux temps : le temps de la rationalité économique où seules les considérations d'efficience interviendraient, et où donc il s'agirait de mesurer de façon objective la productivité d'une personne, sans considération de justice; le temps des politiques publiques d'insertion ou de redistribution qui corrigeraient ces évaluations économiques pour des considérations de justice sociale. L'évaluation, comme nous allons le montrer dans cet article, articule étroitement ces deux temps. ${ }^{1}$

\footnotetext{
${ }^{1}$ Cet article s'appuie sur une série de travaux menés au Centre d'études de l'emploi et à l'université Paris X : Eymard-Duvernay, Marchal, 1997 ; Bessy, Eymard-Duvernay (sous la dir.) (1997) ; Bessy et alii (sous la dir.) (2001). Il doit beaucoup également à une collaboration de long terme avec l'association d'insertion Transfer. Des versions antérieures et partielles ont fait l'objet de présentations dans différents cadres : Les cinquièmes entretiens de l'emploi, organisés par l'ANPE en octobre 2007 ; le séminaire "Quand les entreprises recrutent».
}

* François Eymard-Duvernay est économiste, professeur à l'université Paris X (EconomiX). II est notamment l'auteur de : Façons de recruter, le jugement des compétences sur le marché du travail lavec Emmanuelle Marchal, Métailié, 1997); Économie politique de l'entreprise (La Découverte, 2004). II a dirigé l'ouvrage L'économie des conventions, méthodes et résultats (tomes 1 et 2, La Découverte, 2006). Il a dirigé la publication de : Les intermédiaires du marché du travail (avec Christian Bessy, PUF, 1997). Des marchés du travail équitables? Approche comparative France/RU lavec Christian Bessy, Guillemette de Larquier, Emmanuelle Marchal, PIE Peter Lang, 20011.

\begin{abstract}
Présentation des résultats de l'enquête «Offre d'emploi et recrutement », organisé par la DARES en novembre 2007 ; le séminaire « Lecture institutionnaliste de la RSE », organisé par l'université Paris 12 (IRG) avec le concours de la chaire de développement durable de Sciences Po Paris, en novembre 2007; le séminaire « 20 ans d'expérience IOD : quelles perspectives ? », organisé par l'association Transfer en novembre 2007. Francis Valls, lors de ce dernier séminaire, a opportunément évoqué le titre de l'une des publications qui a initié le programme « Économie des conventions » : Justesse et justice dans le travail, sous la dir. de Luc Boltanski et Laurent Thévenot, 1989, ce qui m'a donné l'idée de le reprendre ici.
\end{abstract}


L'analyse développée met l'accent sur les problèmes d'évaluation lors des recrutements. Dans la première partie, nous montrons que la mesure de la compétence est conditionnée par des principes d'évaluation, qui intègrent des valeurs. Dans la seconde partie, nous introduisons la notion de convention de qualité, comme accord sur un principe d'évaluation. Nous insistons sur la pluralité des conventions, et le débat qui en résulte. Dans la troisième partie, nous développons l'approche suivie à partir d'études de cas et nous présentons plusieurs travaux empiriques en cours mobilisant les annonces d'offres d'emploi. Dans la quatrième partie, nous reprenons le thème fort débattu aujourd'hui de la diversité des recrutements. Plus largement, nous esquissons les conditions d'une évaluation équitable et les dispositifs de recrutement qui en découlent.

\section{DE LA MESURE DES COMPÉTENCES À LA PLURALITÉ DES PRINCIPES D'ÉVALUATION}

Dans les travaux économiques, il est habituel de supposer que les personnes recrutées sont dotées d'une productivité ; l'évaluation est donc sa mesure. Elle est soumise à des problèmes d'erreurs de mesure, comme tout élément de la nature l'est au regard des sciences physiques. La réalité de la compétence est donc supposée extérieure à l'acte de recrutement. L'opération de recrutement occupe d'ailleurs peu de place dans l'analyse économique : dans le schéma classique d'équilibre entre offreurs et demandeurs de travail, il n'y pas d'asymétrie entre évaluateur et évalué, et pas non plus de sélection des candidats à un emploi.

Les approches plus pragmatiques des gestionnaires mettent évidemment au premier plan les questions de sélection et d'évaluation, et ne mentionnent même pas l'asymétrie entre évaluateur et évalué, tellement elle est évidente. Le recrutement y constitue un champ d'analyse à part entière. La question de l'évaluation est, dans ce champ, tendue entre la rationalisation revendiquée par les psychotechniciens et une démarche pragmatique suivie par la plupart des professionnels $\mathrm{du}$ recrutement. La position des psychotechniciens est proche de celle des économistes : il y a une compétence (notion équivalente dans ce champ à celle de productivité pour les économistes), mesurable objectivement par les outils qu'ils développent. Les professionnels pragmatiques travaillent avec des critères d'évaluation qu'ils construisent au coup par coup en fonction des postes à pourvoir, et en mobilisant une gamme souple d'outils, ou alors s'en remettent à leur intuition. La question de l'entretien de recrutement divise profondément ces deux groupes. Pour les psychotechniciens, l'entretien informel est entaché d'arbitraire et plusieurs études montrent que sa prédictivité sur la compétence serait faible (Levy-Leboyer, 1990) ; pour les professionnels pragmatiques, c'est là que se forme le jugement.

Ces deux positions polaires, le rationalisme des économistes et des psychotechniciens d'un côté, le pragmatisme des professionnels de l'autre, sont insatisfaisantes. Nous proposons une reformulation assez profonde de la question de l'évaluation. La novation principale consiste à introduire, dans l'évaluation, deux niveaux : celui de la mesure et celui des principes de mesure. L'évaluation est conditionnée par une opération préalable: l'arbitrage entre plusieurs principes de mesure. Il ne relève pas de la rationalité, car les différents principes sont incommensurables, il n'y a pas de meta unité de mesure qui les transcende. Le pragmatisme des acteurs du recrutement opère en fait implicitement cet arbitrage hors calcul. Il importe de mieux l'expliciter, car le choix d'un principe d'évaluation est incrusté dans toute la chaîne d'opérations.

La notion de principe suggère une cohérence plus globale que le choix de tel ou tel critère : il y a une pluralité de principes, sinon le second niveau n'a pas d'intérêt, mais non une infinité, sinon la notion même de principe perd de son intérêt... Elle suggère également que des valeurs sont en jeu : les principes induisent une dimension morale, absente aussi bien de la rationalité que de l'approche pragmatique. Le fait que des valeurs soient mobilisées dans l'évaluation ne devrait pas surprendre. Nous mettons en scène ainsi un évaluateur réflexif, qui ne fait pas que mettre en œuvre des outils de mesure, mais qui s'interroge sur les principes qui les fondent. Bien entendu, il a des plages de repos importantes, 
lorsqu'il se laisse porter par la routine des outils. Mais son sens critique demeure toujours en éveil, d'autant plus que chaque évaluation a des enjeux propres lourds de conséquences pour différentes personnes.

Il y a trois types de conditions pour que l'on puisse parler de valeurs. En premier lieu, les valeurs doivent résulter d'une construction collective: ceci exclut aussi bien les préférences subjectives, qui n'ont aucune dimension collective, que les grandeurs naturelles, qui n'ont aucune dimension sociale. En second lieu, la notion de valeur comporte l'idée de pluralité et d'incommensurabilité entre des valeurs différentes. S'il y a un système de valeurs unique et cohérent, la notion de valeur perd de son intérêt: les grandeurs sont assimilables à des grandeurs naturelles. En troisième lieu, et ceci découle pour partie du point précédent, la notion de valeur suppose une certaine réflexivité dans le comportement des individus : on ne suit pas des valeurs de façon mécanique.

Soit la scène suivante du film de Wim Wenders, Les ailes $d u$ désir. L'ange Damiel entre dans le petit cirque où Marion s'entraîne sur son trapèze, tout de blanc vêtue avec des ailes dans le dos. L'entraîneur la critique : «Marion, pas comme ça. Avec élan, pas avec force. Ne pendouille pas, vole. Tu es un ange». Marion s'énerve en expliquant que les ailes la gênent. L'entraîneur: "concentre-toi, fais un effort ». Marion : «si je ne faisais pas un effort, je vous serais déjà tombée sur la tête »... Finalement, quelqu'un accourre: "Arrêtez tout, on ne peut plus payer ; pour cette année, le cirque est mort. »

On voit en premier lieu, dans cette petite scène, que la compétence s'évalue dans un débat critique. On ne peut observer la compétence au repos, comme semblent parfois le penser les psychotechniciens. En second lieu, on voit que l'évaluation passe par un langage finement modulé : "avec élan, pas avec force; ne pendouille pas, vole». Le maniement complexe du langage permet de prospecter plusieurs espaces de valeur, afin de mieux faire ressortir la valeur qui vaut vraiment, synthétisée dans l'expression: «Tu es un ange ». L'évaluation a une dimension qualitative importante, du fait de cette prospection des valeurs, le quantitatif n'intervenant qu'une fois les principes de qualité fixés (on doit même pouvoir mesurer les performances sur l'échelle « ange »), mais ils ne le sont jamais définitivement. L'incertitude qualitative exprime cette activité primordiale de prospection des qualités. Enfin, il apparaît une issue brutale au débat: l'activité est condamnée par le verdict du marché ; il n'y a plus d'argent. Il y a un changement d'échelle dans l'évaluation: ce n'est plus la compétence au trapèze de Marion qui est en jeu, mais plus globalement le cirque, sans doute lui-même pris dans un contexte plus général de crise. On pourrait penser que tel est le bon niveau d'analyse (le « macro ») et que le reste est anecdotique. Mais on ne peut cliver ainsi le « macro » du « micro » : il n'y a pas d'espace surplombant qui verrouillerait ex ante les évaluations.

Prenons une source plus académique. Gautié et alii (2005) ont observé un chasseur de tête au cours d'une mission pour une entreprise du secteur «du luxe», restructurée par un grand cabinet de conseil en stratégie. Nous reprenons de brefs extraits de cette observation. «On nous demandait de rechercher des profils qui n'existent pas. Ils ont inventé des noms de postes très pompeux, complexes et tendance mais nous, je ne vois pas ce qu'on peut faire avec ça. » Ou encore: «En fait ce qu'ils voulaient c'était un bon directeur des achats et non un machin chose délirant. Le cabinet de stratégie X est passé avant et nous on décrypte par rapport à ce que l'on peut connaitre des besoins de l'entreprise et de la réalité du marché. » (p. 389) Cette observation montre bien la tension entre plusieurs valeurs qui s'expriment dans les intitulés d'emploi : des noms de poste «pompeux, complexes et tendance » versus « directeur des achats ». Le chasseur de tête dénonce les valeurs prônées par ses collègues du cabinet de conseils en stratégie au nom de la « réalité du marché ». On peut concevoir pourtant que des innovations dans les biens commercialisés induisent des innovations dans les valeurs qui soutiennent les compétences. Le rabattement sur « la réalité » est une façon classique d'éliminer le débat sur les valeurs par une posture rationaliste. On oublie que « directeur des achats » relève de valeurs, qui n'apparaissent plus comme telles parce qu'elles sont routinisées. Notre objectif n'est évidemment pas d'adopter une posture anti-rationaliste, mais de faire reconnaître le débat sur les valeurs, qui relève d'une rationalité élargie, non réductible au calcul. 
Nous développerons d'abord au plan théorique cette approche de l'évaluation pour ensuite l'illustrer à partir d'études de cas et de travaux empiriques.

\section{LE DÉBAT SUR LES CONVENTIONS DE QUALITÉ}

L'approche conventionnaliste (voir encadré 1) a deux spécificités. Le bien commun, qui sous-tend l'évaluation, résulte d'un accord entre les personnes sur la finalité commune de leur action : par exemple le bien à fabriquer et commercialiser pour une entreprise $^{2}$. C'est une approche de la coordination en termes de contrat social qui est ainsi privilégiée. La

\footnotetext{
${ }^{2}$ Nous utilisons la polysémie du mot «bien » : il désigne l'objet concret à fabriquer, mais aussi et indissociablement une valeur qui s'inscrit dans un projet de vie (j'achète une montre pour ne plus être en retard), soit une valeur morale.
}

visée d'un accord sur le bien commun peut paraître trop exigeante. Il suffit en fait de supposer un accord sur le cadre de débat sur le bien commun, soit un accord sur la façon de régler les désaccords. Deuxièmement, il y a un arbitrage à réaliser entre plusieurs conventions possibles. Les conventions qui soutiennent la coordination ne sont pas des dispositions solidement ancrées dans les agents, comme les habitus ou les cultures : les agents sont capables de changer de convention lorsque la situation de coordination l'exige. Elles ne sont pas non plus des institutions hors d'atteinte des agents : ils ont une capacité de les modifier, lorsque cela apparaît nécessaire. Les conventions sont implantées dans des objets (biens fabriqués et commercialisés, équipements techniques, etc.), dans le langage (qui permet la diffusion de l'information et incorpore des conceptions du bien), dans des formes instituées telles que les règles de droit. Ces dispositifs stabilisent les conventions, mais sont aussi des leviers pour les modifier.

\section{Encadré 1 \\ Le programme de recherche sur l'Économie des conventions}

Ce courant de recherche, initié dans le numéro spécial de la Revue Économique " L'économie des conventions » (mars 1989), présente deux critiques majeures à la théorie néoclassique :

Cette dernière est fondée sur le postulat d'une autonomie complète de l'individu, exprimée par l'hypothèse de rationalité substantielle et par la figure du contrat entre individus libres et égaux. Pour sa part, l'économie des conventions met l'accent sur la nécessité d'un cadre commun pour soutenir l'accord entre les individus, c'està-dire d'une convention constitutive.

À l'encontre de la tentative d'extension du marché à l'ensemble des relations sociales portée par la théorie moderne des contrats, l'Économie des conventions introduit une pluralité de formes de coordination. Elle prend appui sur les modes de coordination travaillés par les autres sciences sociales, sans les faire rentrer à peu de frais dans le modèle de marché $(*)$.

L'ambition du nouveau paradigme des contrats (soit la "Théorie standard étendue » suivant l'expression d'Olivier Favereau), qui vise en fait une extension à l'ensemble des sciences sociales, nécessiterait une refonte profonde des méthodes d'analyse. La théorie standard étendue fonde sa construction sur une axiomatique de la rationalité pertinente dans l'approche classique du marché, mais qui est irréaliste dans les nouvelles approches, et qui a été largement infirmée par l'observation. Elle ne tire pas toutes les conclusions des apories mises en lumière par la théorie des jeux. Elle reformule abusivement dans le langage unique du marché étendu aux contrats des relations dont l'observation montre qu'elles relèvent d'autres grammaires.

L'originalité de l'Économie des conventions est d'établir une connexion étroite entre des approches économiques et des approches sociologiques. Pour cette dernière discipline, l'un des appuis importants est le "modèle des économies de la grandeur» (Boltanski, Thévenot, 1991 ; Boltanski, Chiapello, 1999). 


\section{Encadré 1 (suite)}

Ce modèle analyse la façon dont les personnes, pour régler leurs différends, soumettent leurs actions et celles des autres à des principes de justification généraux.

Il prend appui sur des philosophies politiques pour établir des systèmes de contraintes auxquels les individus sont soumis lorsqu'ils tentent de justifier leurs actions. Les philosophies politiques sont des formes systématisées de grammaires du lien politique, c'està-dire de contraintes pour qu'un ensemble de personnes forme une Cité.

Six Cités ont ainsi été identifiées par les auteurs comme jouant un rôle majeur dans les sociétés contemporaines : les cités marchande, industrielle, domestique, civique, du renom, inspirée. L. Boltanski et E. Chiapello (1999) ont analysé l'émergence d'une nouvelle cité (la cité par projets) dans les trente dernières années.

La question de la coordination est rapportée à celle de l'accord sur l'ordre entre les personnes dans une situation donnée. Les "épreuves » (un examen scolaire, un recrutement, un moment de travail où la compétence est mise à l'épreuve, etc.) permettent de lever l'incertitude sur cet ordre en qualifiant les personnes en référence à des principes de justice. Les épreuves mettent en jeu des critiques et des justifications : un salarié estime être injustement évalué, son supérieur hiérarchique argumente en réponse à ces critiques, etc. La pluralité des ordres de grandeur rend difficile la convergence du jugement, un nouveau point de vue étant toujours susceptible de faire reconsidérer complètement ce qui paraissait bien établi.

(*) Le lecteur qui souhaiterait des approfondissements peut se référer à l'une des publications à vocation synthétique qui ont jalonné ce parcours (on trouvera sur le site de Capitalismes et Démocraties un ensemble de références du programme « conventions », ainsi qu'un échantillon de textes en ligne) : L'économie des conventions (numéro spécial de la Revue économique, sous la dir. de F. Eymard-Duvernay, mars 1989); Analyse économique des conventions (sous la direction de A. Orléan, PUF 1994, réédité en 2004) ; Institutions et conventions. La réflexivité de l'action économique (sous la direction de R. Salais, E. Chatel, D. Rivaud-Danset, Éditions de l'École des hautes études en sciences sociales, 1998), Théorie des conventions (sous la direction de P. Batifoulier, Économica, 2001), Conventions and Structures in Economic Organization (Edited by O. Favereau and E. Lazega); L'économie des conventions, méthodes et résultats. Tome 1 Débats, Tome 2 Développements (La Découverte, collection « Recherches », 2006).

Né en France, ce courant de recherche entretient des coopérations internationales : le Cambridge realist workshop (Professor Tony Lawson) ; les universités de Cambridge (Simon Deakin) et Essex (S. Leader) ; le réseau européen EUROCAP, coordonné par Robert Salais (IDHE Cachan); la filière institutionnaliste de la «High School of Economy» de l'université de Moscou (Professor Oleg Ananyine) ; le département de sciences sociales de l'université de Louvain La Neuve (Professeur Jean De Munck) ; des personnalités mondialement reconnues: Michael Piore (MIT), David Marsden (LSE), Harisson White (Columbia University), David Stark (Columbia University), Richard Swedberg (Cornell University), etc.

Les deux présupposés ainsi exposés sont en tension. En effet, la pluralité des conventions fragilise l'accord sur un bien commun. Le modèle des Cités (Boltanski, Thévenot, 1991; Boltanski, Chiapello, 1999, cf. encadré 1), met ainsi au premier plan les opérations de critique, plutôt que l'accord stabilisé sur un cadre d'évaluation. L'hypothèse minimale mobilisée est celle de réflexivité : les acteurs ne suivent pas de façon routinisée un cadre d'évaluation, mais en débattent.

Des conventions particulièrement cruciales sont celles qui soutiennent l'évaluation des biens et du travail, que l'on nomme conventions de qualité; ce sont des principes d'évaluation sur lesquels il y a un accord. De nombreux travaux d'économie de l'information montrent bien que la coordination devient problématique dans les situations d'incertitude qualitative (Akerlof, 1970 ; Stiglitz, 1987 ; Orléan, 1991). Le rôle des conventions de qualité n'est pas envisagé par l'économie standard, parce qu'il n'y a pas plusieurs principes de qualité dans ce paradigme. La qualité est supposée exister préalablement à tout échange : c'est «l'hypothèse de nomenclature » (Benetti, Cartelier, 1980), ou l'introduction d'une variable «qualité», supposée dotée d'une mesure préalable, dans l'économie de la qualité. Ou alors, le marché est considéré comme le seul dispositif légitime d'évaluation de la qualité. Mais cette dernière hypothèse relègue au second plan les entreprises comme dispositifs spécifiques d'évaluation de la qualité. 
Une fois les conventions de qualité fixées, le calcul rationnel peut se dérouler sans problème, de même que lorsque les unités de compte sont fixées. Mais il est nécessaire d'endogénéiser, à l'analyse, leur construction, car elles ne sont jamais définitivement établies mais remises en cause continûment dans le cours de la coordination. Ce débat sur les conventions, qui sous-tend la coordination, est pris en compte dans l'économie des conventions par l'introduction d'une pluralité de conventions entre lesquelles les acteurs ont à arbitrer. L'outil d'analyse a la forme d'une typologie de conventions. Il faut préciser que cette typologie est une formalisation des opérations réalisées par les acteurs lorsqu'ils débattent des conventions à suivre, et non une cartographie statique d'un espace qui serait partitionné entre différentes conventions. Elle dessine plutôt un espace de controverses dans les situations où les acteurs discutent pour établir une convention. D'un point de vue méthodologique, elle est établie en s'appuyant sur la confrontation entre différentes théories sur la société, développées en philosophie politique ou dans les sciences sociales, avec l'hypothèse que les acteurs mobilisent de façon informelle une controverse explicitée et formalisée par ces disciplines. Nous l'illustrerons dans la partie suivante à partir d'un exemple.

Cette méthodologie a été introduite par L. Boltanski et L. Thévenot (1991), qui prenaient appui sur des philosophies politiques pour caractériser chaque «cité », en les relayant par des manuels de gestion afin de repérer les grammaires propres à chacune (Boltanski, Chiapello, 1999). Pour des travaux sur le recrutement, nous avons prolongé cette démarche afin de dresser une typologie de conventions de qualité du travail (ou conventions de compétence ${ }^{3}$ ) (Eymard-Duvernay, Marchal, 1997；2000). Mais, d'une part, notre appui était constitué par des

\footnotetext{
${ }^{3}$ Nous utilisons ici la notion de compétence de façon générique, comme synonyme de qualité du travail. Le terme «logique compétence » est, dans la période récente, souvent utilisé pour désigner une acception particulière de la qualité du travail. La « logique compétence» est ainsi, dans notre terminologie, une convention de compétence particulière.
}

modèles de sciences sociales et non des philosophies politiques; d'autre part, nous avons organisé la controverse entre les différentes conventions, alors que les cités sont considérées comme des registres d'évaluation incommensurables. Nous avons organisé cette controverse autour de deux axes de débats : l'un opposant interprétations planifiée et négociée des compétences, l'autre interprétations individualiste et sociologique des compétences. Sans entrer dans une discussion de fond, il nous semble que cette méthode permet de mieux rendre compte de la façon dont le débat sur les conventions se stabilise sur un « équilibre » entre les différentes conventions en jeu, et non sur une seule convention dont la logique serait poussée à terme (processus de «montée en généralité » du modèle des cités).

Le débat sur les conventions fait naturellement intervenir des questions de justice : comment les bénéfices de la coopération sont-ils répartis entre les différentes parties? Elles interviennent dès le stade de l'évaluation, qui est soumise à des contraintes d'objectivité (registre cognitif) et de justice (registre politique). Pour ce qui concerne l'évaluation de la qualité du travail, on ne peut supposer, comme nous l'avons déjà dit, qu'il y aurait d'abord une phase d'évaluation, gouvernée uniquement par des considérations d'objectivité, puis une phase de redistribution, gouvernée par des considérations de justice. Les théories de la justice permettent d'analyser les contraintes que doivent respecter les évaluations pour être considérées comme justes (Rawls, 1987), et les dispositifs associés. La discrimination est naturellement le comportement le plus extrême, mais des comportements peuvent ne pas relever des critères discriminatoires sans pour autant être équitables. C'est le cas par exemple lorsque le diplôme a un poids excessif dans l'évaluation, conduisant à la sursélection; ou lorsque le candidat à un emploi est évalué comme un objet, sans pouvoir argumenter luimême sur sa compétence. Ces questions sont approfondies dans la quatrième partie.

Dans la partie suivante, nous explorons les opérations d'évaluation de façon moins abstraite, en nous appuyant sur des études de cas; nous montrons également quels types de données statistiques peuvent être construits pour repérer les conventions de qualité mobilisées dans les recrutements. 
OBSERVATIONS EMPIRIQUES DE SITUATIONS D'ÉVALUATION : L'INCERTITUDE QUALITATIVE

Soit le cas d'un patron de PME (petite et moyenne entreprise) qui fait appel à un consultant de l'APEC (Agence pour l'emploi des cadres) pour recruter son «bras droit», suivant son expression (EymardDuvernay, Marchal, 1997, ch. 2 ; Eymard-Duvernay, Marchal, 2000). Le consultant utilise l'intermédiaire habituel sur le marché du travail: les annonces d'offres d'emploi. Voici un extrait de l'annonce finalement publiée :

Nous recherchons dans le cadre de notre développement un ADJOINT DE DIRECTION. Sa mission: véritable bras droit de la Direction Générale, il aura à superviser l'ensemble du personnel des services suivants: comptabilité, administratif, achats, logistique, administration des ventes. Nous recherchons pour ce poste un candidat de 35/45 ans environ, de formation bac +2 minimum et ayant une expérience similaire au sein d'une PME du secteur du négoce.

S'il a repris, un peu par courtoisie vis-à-vis de l'employeur, l'expression « bras droit », le consultant n'a pu évidemment s'en tenir à une telle qualification pour rédiger son annonce et a dû décomposer les tâches à réaliser afin de retraduire l'emploi : il faut rendre compatible le codage et le canal de circulation de l'information, mettre les connaissances dans le format qui leur permette de circuler sur un canal d'informations, ici les petites annonces. Le consultant a mis en avant la qualification d'adjoint de direction et a présenté l'entreprise en termes de services, conformément aux standards en vigueur sur le marché du travail et dans les grandes organisations. $\mathrm{Au}$ final, il a fait figurer un critère de diplôme, qui n'était nullement mis en avant par l'employeur. Du « bras droit » à « bac +2 », le langage de désignation de l'emploi a pour le moins évolué sous les contraintes de rédaction de l'annonce.

On peut interpréter l'opération réalisée par le consultant en termes de rationalisation de la demande de l'employeur. Mais cette interprétation est trop unidimensionnelle: elle repose sur une opposition binaire entre l'informel (bras droit) et le formel $(\mathrm{bac}+2)$, le spécifique et le général, le qualitatif et le quantitatif, etc. Une approche plus ouverte montre qu'il y a une pluralité de façons de formaliser, de coder les connaissances pour les faire circuler dans des réseaux de communication. « Bras droit » n'est-il pas lui-même un langage codé, dans un certain registre? Cette ouverture permet de montrer que le consultant a fait une opération de traduction d'un langage à un autre, ce qui change complètement l'interprétation de son rôle. Elle permet en particulier l'exercice de la critique : pourquoi le consultant ne s'en est-il pas tenu au registre spontané de l'employeur (le registre domestique dans le langage du modèle des cités ${ }^{4}$ ? Cette question est d'autant plus pertinente que les conséquences en termes de sélection sont lourdes : ont été éliminés d'emblée les non-diplômés, alors que cela ne semblait pas être une contrainte cruciale de l'emploi. On voit à ce propos que la situation de chômage est étroitement liée à ces opérations d'évaluation. Les modes d'évaluation induisent en effet les qualités qui seront valorisées par l'embauche ou au contraire rejetées dans le chômage. Ils sont également plus ou moins sélectifs, donc rejettent au chômage une proportion plus ou moins grande de la population 5 (Eymard-Duvernay, 2005).

Pour progresser dans l'analyse, il faut décomposer plus finement les opérations réalisées par le consultant pour la rédaction de l'annonce, puis la sélection des candidatures. Comment a-t-il établi les critères qui figurent dans l'annonce? Ces critères n'ont rien d'original, ils sont conformes aux standards du marché du travail, ce qui est déjà une information importante. Mais, malgré tout, il n'a pas suffi d'activer mécaniquement ces standards : il a fallu opérer des réglages.

\footnotetext{
${ }^{4}$ Le registre domestique, dans le modèle des Économies de la grandeur (Boltanski, Thévenot, 1991), valorise les relations de proximité sur le long terme, la confiance entre un supérieur hiérarchique et les membres de son équipe, l'engagement personnel dans le travail, l'absence de clivage entre relations professionnelles et relations personnelles. Les biens correspondant sont des biens de qualité traditionnelle, garantie par une maison ancienne, une appartenance locale.

${ }^{5}$ Par exemple, exiger « bac +2 » rejette dans le chômage plus de personnes que si l'emploi ne présente pas d'exigence de diplôme.
} 
Soit l'exemple de l'âge. Le raisonnement du consultant est le suivant : le patron a la cinquantaine, il faut donc quelqu'un d'un peu moins âgé, afin qu'il garde son autorité. Ce raisonnement est une interprétation d'une théorie de la coordination, que l'on pourrait appeler démographique: l'exercice de l'autorité s'évalue à l'âge. Stupide ? Ce qui nous importe à ce stade, c'est que le consultant mobilise une théorie de la coordination : pour qu'un patron et son adjoint se coordonnent bien, c'est-à-dire que chacun fasse ce qui est attendu par l'autre sans disputes récurrentes, il faut que le premier soit plus âgé que le second.

Autre raisonnement concernant le sexe : il y a une secrétaire de direction femme, donc il vaut mieux recruter un homme pour éviter les disputes. Là aussi une théorie de la coordination, fondée sur les rapports entre les sexes. Encore plus stupide?

Que le recruteur fixe comme critère une expérience analogue dans une PME du commerce heurte moins. Pourtant, c'est bien encore une théorie de la coordination: les personnes, pour s'ajuster à leur environnement, doivent posséder des compétences conformes à la culture d'entreprise. Cette interprétation a ses lettres de noblesse dans les sciences sociales : les approches culturalistes sont bien documentées pour analyser les entreprises. Pourtant l'élimination par le consultant, dans son tri de candidatures, de salariés venant de banques ou d'institutions sociales, scolaires, médicales, peut prêter à discussion: y a-t-il une telle force des cultures d'entreprise qu'elles imprègnent de façon irréversible la compétence?

La référence au diplôme ne choque en aucune façon le lecteur français ; pourtant, elle serait plus suspecte au lecteur anglais, dans la mesure où dans son pays le critère du diplôme est beaucoup moins mobilisé.

Première conclusion de cette observation : les évaluateurs doivent mobiliser des théories de la coordination pour évaluer la compétence. C'est tout à fait compréhensible: la compétence est relative à une situation de coordination donnée. Ces théories sont liées à des principes de qualité. Il y a une incertitude qualitative : c'est l'incertitude sur la bonne « théorie » de la coordination, qui conditionne la façon d'évaluer la compétence. Nous parlerons aussi d'incertitude épistémique (Orléan, 1991), pour faire le rapproche- ment avec la situation des chercheurs qui s'interrogent sur la validité d'une théorie scientifique.

Dans la théorie économique standard, il y a deux niveaux dans l'analyse :

- Des qualités naturelles (force physique, capacité cognitive, réflexes, traits de caractère, etc.) ou socialement acquises (diplôme, expérience, etc.) ;

- Des mesures qui traduisent ces qualités en données quantifiées.

Nous introduisons un niveau supplémentaire pour rendre compte de l'évaluation: les théories de la coordination. Il est un préalable à l'identification des qualités qui doivent être prises en compte pour évaluer la compétence.

- Théories de la coordination des acteurs. Ainsi, la qualification de «bras droit» (qui renvoie à une coordination par relations interpersonnelles) et la qualification « d'adjoint de direction » (qui renvoie à une coordination bureaucratique) conduisent à des exigences de diplôme très différentes: pas d'exigence dans le premier cas, bac +2 dans le second ;

- Qualités naturelles ou socialement acquises dans le format de ces théories ;

- Mesures qui traduisent ces qualités en données quantifiées.

Cette complexification n'a d'intérêt que s'il y a plusieurs théories en concurrence : il faut alors un arbitrage sur la bonne théorie de la coordination. Avant d'évaluer la compétence, il faut évaluer les théories de la coordination. Cette incertitude est plus profonde que l'incertitude probabiliste ${ }^{6}$, c'est l'incertitude épistémique.

Nous devons maintenant aborder une seconde question issue de notre observation : toutes les théories sont-elles acceptables pour le chercheur, peut-il les classer? Ce point de vue du chercheur peut-il correspondre à celui des acteurs? Nous avons vu que certaines théories paraissaient intuitivement illégitimes (évidemment cela aurait été encore pire si notre

\footnotetext{
${ }^{6}$ Nous utilisons le terme incertitude sans faire la distinction, introduite par Knight, entre risque et incertitude. Cette distinction est essentielle. Mais elle reste ancrée sur le paradigme probabiliste, en distinguant ce qui est mesurable dans son cadre ou non. Nous mettons ici l'accent sur une autre différenciation des formes d'incertitude : suivant la nature des théories de la coordination que la mesure mobilise.
} 
recruteur avait mobilisé l'astrologie!), mais que d'autres passaient mieux auprès du chercheur. Le partage semble être entre celles qui ont droit de cité dans les sciences sociales et les autres. Ce partage est compréhensible puisqu'il s'agit d'identifier des théories de la coordination et que c'est la spécialité des sciences sociales. On peut, comme nous l'avons vu précédemment, rapprocher les théories de la coordination des théories développées par les sciences sociales, qui sont ainsi supposées faire de façon systématisée des opérations réalisées de façon plus informelle par les acteurs dits ordinaires. On peut ainsi poser l'hypothèse que le sens de la justice du chercheur, équipé par les sciences sociales, correspond au sens de la justice des acteurs « ordinaires » : les théories des sciences sociales ne font qu'expliciter les jugements de ces derniers.

$\mathrm{S}$ 'en tenir à ces théories permet de sélectionner celles qui ont un minimum de légitimité. Le chercheur peut dresser une carte de controverses entre elles et observer comment les acteurs font des arbitrages ou des compromis. Dans cette méthode, l'observation se focalise sur les dispositifs d'évaluation (évaluation en face-à-face au cours d'un entretien, ou sur la base de CV, par la prise en compte du diplôme ou de propriétés psychologiques, etc.). L'observation doit s'affiner pour prendre en compte des réglages fins des dispositifs. Ainsi, des observations portant sur des associations qui soutiennent l'insertion de chômeurs de longue durée (en particulier l'association Transfer: Castra, Valls, 2007) montrent que suivant le lieu des entretiens d'évaluation (chez l'employeur potentiel ou au siège de l'association), suivant les personnes présentes (présence ou non du chargé de mission de l'association, des collègues de travail du poste à pourvoir, etc.), l'évaluation sera plus ou moins sélective. Plus l'évaluation est planifiée, sur des critères de pré-jugement généraux, plus elle entretient le chômage de longue durée (Salognon, 2005).

L'observateur doit également être attentif au vocabulaire mobilisé par les personnes pour se coordonner: différents registres de vocabulaires permettent très simplement de repérer différentes théories de la coordination implicitement mobilisées. Cette méthode a été instrumentée, dans une perspective très large, par un logiciel de traitement de données textuelles, Prospero, développé sous la direction de F. Chateauraynaud (voir le cadre conceptuel général de cet auteur, 2003).

Les annonces d'offres d'emploi ont été analysées avec cet outil (Marchal, Torny, 2003; Marchal, Rieucau, 2006; Marchal, Mellet, Rieucau, 2007), qu'il s'agisse des annonces papier ou des annonces sur internet. Ce matériel textuel présente le grand avantage d'être un maillon d'une chaîne de relations opérationnelles : il permet donc de repérer le langage effectivement choisi pour la coordination. On pourra objecter à ce support que les annonces ne constituent qu'un intermédiaire parmi d'autres des mises en relation, et d'importance numérique assez faible. Néanmoins, elles représentent la partie publique du marché du travail et à ce titre révèlent les concepts qui ont le plus de légitimité sur ce marché, au-delà des intermédiations qui transitent effectivement par des annonces. Les annonces révèlent en effet les standards d'évaluation des recruteurs, sans doute activés dans d'autres situations. Malgré tout, il serait abusif de généraliser complètement les informations de ce mode de recrutement: les canaux de recrutement et leurs contraintes spécifiques influent sur le jugement.

L'exploitation de ces données fournit des informations originales sur l'évolution en longue période des critères d'évaluation (Marchal, Rieucau, 2006). On constate ainsi l'augmentation spectaculaire, entre 1960 et 1990, en France, des exigences de formation et d'expérience, ainsi que la montée des références à des qualités personnelles. Cette évolution traduit, outre l'élévation du niveau d'éducation, la sélection liée à la masse de candidatures disponibles, ainsi que la sous-traitance des recrutements à des cabinets privés de professionnels du recrutement. Il est intéressant de constater également le déclin des annonces sexuées, qui montre la diffusion en France, tardive il faut bien le dire, des préoccupations concernant la discrimination suivant le genre. En revanche, la citation de l'âge dans les critères de recrutement ne diminue pas. Au total, il apparaît une sélection accrue des candidats sur des critères généraux, de nature à expliquer qu'une part importante de chômeurs ne parvienne jamais au stade de l'entretien. Plus les annoncent présélectionnent, moins ont de chance de parvenir à l'entretien ceux qui n'entrent pas dans les critères. Même si les critères utilisés ne 
sont pas juridiquement discriminatoires, il s'agit là d'une forme de "pré-jugement» (suivant l'expression contenue dans le titre de Marchal, Rieucau, 2006) qui s'apparente à de la discrimination.

L'exploitation des données sur des annonces anglaises, espagnoles et françaises permet une approche comparative (Marchal, Rieucau, 2006). On constate ainsi le poids considérable du critère de formation en France. Cette situation n'est pas critiquable en elle-même. L'investissement public dans l'École comme moyen de promotion sociale est l'une des options majeures de la République française. Par ailleurs, la négociation collective s'est développée en associant des postes à des niveaux de formation. Mais on sait bien que les conditions ont changé : l'inégalité accrue d'accès aux diplômes fragilise la légitimité de ce critère. On peut penser que le recours généralisé au niveau de formation comme critère de recrutement traduit non des exigences par rapport à des nécessités concrètes des emplois, mais la recherche de «potentiels» dans une situation de forte incertitude. Y a-t-il toujours, comme l'exige la loi, un «lien direct et nécessaire avec l'emploi proposé »? Les annonces espagnoles ont, elles, outre la référence au genre et à l'âge, la particularité d'exiger fréquemment un ancrage régional des candidats. On constate de façon générale que les annonces britanniques sont les plus légères en termes de critères de présélection, et les plus rigoureuses dans l'abstention par rapport aux critères discriminatoires.

L'approche comparative internationale s'est développée et renouvelée, en particulier sous l'impulsion des travaux novateurs de "l'approche sociétale». (Maurice, Sellier, Silvestre, 1982). À côté de travaux statistiques globaux, où chaque pays est un point caractérisé par une batterie de variables, il apparaît fructueux de développer des analyses focalisées sur des institutions précises qui permettent de comprendre plus en profondeur la façon dont les compromis sociaux se nouent dans les différentes économies. Ces investigations d'apparence locale sont riches d'enseignements globaux : le «macro» est inscrit dans le « micro ». Le recrutement constitue à cet égard un point d'observation très intéressant, complètement négligé. Les modes d'évaluation tels qu'ils sont observables au niveau micro dans les recrutements sont en effet révélateurs des ordres sociaux qui structurent une économie. La comparaison entre la France et le Royaume-Uni est de ce point de vue instructive (Bessy et alii, 2001). L'opposition entre un marché libre et une économie dirigée par l'État, souvent mobilisée pour la comparaison entre ces deux économies, y apparaît insuffisante: on sait que les agences publiques de placement se sont développées très précocement en Angleterre sous l'impulsion de Beveridge, et que par ailleurs la législation anti-discrimination y est beaucoup plus présente qu'en France. La comparaison précise des institutions du recrutement montre que le marché anglais est plus équipé que le marché français. On peut même parler, pour cette économie, d'une véritable industrie du recrutement, avec ses intermédiaires publics et privés qui forment un maillage serré du marché du travail et ses outils d'évaluation, en particulier les tests soutenus par une forte corporation de psychologues du travail. Les approches économiques qui contrastent ces deux économies à partir de l'opposition entre marché externe du travail (GrandeBretagne) et marchés internes ${ }^{7}$ (France) sont justes. Mais il faut aller au-delà d'une caractérisation de ces deux types de marchés en termes de flux d'emplois entre les entreprises et le marché. L'observation précise des modes de recrutement montre des différences importantes sur les modes d'évaluation.

\section{LA DIVERSIFICATION DES RECRUTEMENTS : POUR UNE ÉVALUATION ÉQUITABLE}

La question de la diversification des recrutements devient un thème majeur en France. On peut l'envisager dans un cadre plus général que celui de la discrimination. Il s'agit de réfléchir aux comportements d'exclusion, non seulement de catégories protégées par la loi anti-discrimination, mais plus généralement de «minorités », au sens de personnes dotées de compétences qui ne sont pas toujours

\footnotetext{
${ }^{7}$ La distinction entre marché externe et marché interne a été introduite et développée par Doeringer et Piore (1971). Alors que, sur le marché externe, le travail est évalué et alloué par les mécanismes du marché, sur le marché interne, il est évalué et alloué par des règles administratives et coutumières. Les salariés font carrière au sein d'un marché interne et y développent des qualifications spécifiques.
} 
visibles dans les référentiels de compétences standards. L'objectif est de lutter contre la «pensée unique » en matière de compétences : faut-il vraiment un bac +2 pour occuper tel emploi ? Est-ce que seul un diplômé de grande école fera l'affaire pour tel autre ?, etc. Au plan social, une sélection plus diversifiée a des conséquences en termes de chômage : les compétences «minoritaires» sont mieux valorisées. Au plan économique, on pressent qu'une équipe de travail « plurielle », mettant en jeu des expériences et des compétences différentes, sera plus efficiente qu'une équipe mono-couleur de compétence. Ariane Ghiradello montre ainsi que les travaux empiriques concluent plutôt, sans que ce résultat soit totalement établi, à un effet positif sur la productivité de l'affirmative action (Ghirardello, 2003, chapitre 5).

Nous proposons à la discussion cinq principes pour améliorer l'équité des recrutements dans cette perspective: un principe de modestie (accepter l'incertitude des évaluations); éviter les risques de sur-sélection; limiter volontairement le pouvoir de l'évaluateur en réduisant l'asymétrie entre évaluateur et évalué; intégrer les aménagements possibles de l'environnement de travail ; pluraliser les valeurs. Ils sont tirés des approches académiques de l'équité, mais aussi de pratiques d'associations d'insertion qui tentent de mettre en œuvre sur le terrain ces principes. Nous avons en particulier étudié les pratiques de l'association Transfer, qui développe la méthode IOD («Intervention sur l'offre et sur la demande »: Castra, Valls, 2007). Le rapport de Gérard LyonCaen sur le recrutement (1992) et les travaux d'Olivier de Schutter sur la discrimination (2001) constituent également des appuis décisifs pour cette démarche.

\section{Un principe de modestie :} accepter l'incertitude des évaluations

L'incertitude radicale qui entoure l'évaluation a plusieurs sources :

- La complexité de la compétence qui ne peut se ramener à quelques aptitudes simples à mesurer ;

- La contextualité de la compétence : la compétence n'est pas une propriété substantielle, elle s'éprouve dans l'appariement à un environnement de travail ;
- La pluralité des critères d'évaluation : il n'y a pas une seule façon d'évaluer la compétence, comme le montrent les comparaisons internationales sur les critères mobilisés.

La méconnaissance de cette incertitude conduit soit à une attitude de sur-rationalisation : multiplication des critères, des épreuves; soit à basculer dans l'irrationnel, ou la pure intuition. Une démarche de rationalité limitée évite ces deux écueils. La posture critique qu'elle comporte induit un effort de rationalisation de l'évaluation: l'évaluateur, attentif aux dérives possibles, s'attache à mieux comprendre et expliciter les procédures qu'il suit. Mais la rationalité limitée bien comprise intègre également les limites de la prédictivité de l'évaluation, ce qui l'empêche de basculer dans la sur-rationalisation. Les épreuves ne doivent pas être disproportionnées par rapport à la précision réelle du résultat.

\section{Éviter le risque de sur-sélection}

Nous avons vu la montée des exigences depuis les années 60 , en termes de diplôme, d'expérience, de qualités personnelles (avec toutes les difficultés d'évaluation que l'on sait). Sont-elles toujours proportionnées aux nécessités de l'emploi ? Ne vontelles pas dans le sens d'une absence de diversité dans l'entreprise ? Que dire de la méfiance à l'égard de ceux qui ont connu une période de chômage (Salognon, 2005)?

Comment, concrètement, éviter les risques de sursélection?

Il importe de mettre l'accent sur les caractéristiques spécifiques de l'emploi et non sur des propriétés générales des candidats. C'est une façon de reconnaître la nature contextuelle de l'évaluation. Cela suppose une description complète de l'emploi (avec indication du salaire, contrairement à ce qui se passe le plus souvent dans les annonces françaises), en ne retenant que les critères de sélection manifestement liés à l'emploi. Au-delà d'une démarche fondée sur des critères, il importe de laisser une place aux interactions au cours desquelles peuvent émerger des compétences non prévues: passer du jugement planifié au jugement négocié. Il peut être utile de confronter les candidats concrètement à l'environnement de travail, et aux collègues de travail. 


\section{Réduire l'asymétrie entre évaluateur et évalué}

L'incertitude de l'évaluation peut induire une fuite en avant dans la sophistication des méthodes d'évaluation : construire un arsenal complet et rigoureux d'outils d'évaluation. Mais cela peut conduire au risque de sur-sélection mentionné. Les pré-jugements des évaluateurs doivent être remis en question (Marchal, Rieucau, 2006). De plus, aussi sophistiquées soient-elles, les méthodes d'évaluation ne suppriment pas l'incertitude radicale.

Une autre voie, plus légère, consiste à limiter le pouvoir de l'évaluateur en le transférant sur l'évalué : du candidat comme objet de l'évaluation au candidat comme sujet de l'évaluation.

Cette méthode a un triple avantage :

- au plan cognitif, elle supplée aux défaillances incontournables de l'évaluateur : finalement, le candidat est le meilleur juge de sa compétence, s'il est bien informé sur l'emploi à pourvoir ;

- Au plan moral, elle contribue au respect des droits des candidats : le droit d'argumenter sur sa compétence, d'être partie-prenante de son évaluation, au lieu d'être un objet de mesure ;

- La prise en compte réelle du point de vue des candidats assure la diversité des recrutements, au contraire des méthodes qui fixent des critères standards et sélectionnent à distance.

Plusieurs conditions doivent être remplies :

- Présenter l'emploi à pourvoir de façon complète et précise, de façon que les candidats puissent se positionner facilement eux-mêmes ;

- Ne pas étendre exagérément l'espace des candidatures : trop d'évaluation tue l'évaluation. Trop de candidats force à se rabattre sur quelques critères simplistes, parfois discriminatoires : diviser une pile de candidatures par deux en se fixant un critère de genre est tentant; procéder à des analyses graphologiques expéditives, etc.;

- Se méfier d'internet et de ses outils de classement automatisés (Mellet, 2006). En effet, il est tentant d'utiliser la puissance des moteurs de recherche pour automatiser la sélection sur quelques critères ;

- L'entretien, si décrié par les psychotechniciens, est un moment crucial de l'évaluation. À condition d'être mené de façon équitable, il est l'occasion pour le candidat d'argumenter de façon détaillée et fine sa candidature, et il donne moins d'importance aux informations qui sautent aux yeux sur un $\mathrm{CV}$ : sexe, âge, trous dans les $\mathrm{CV}$, etc. Il est vrai que ceci demande une compétence spécifique de la part du candidat, d'où l'importance de la recommandation suivante.

- La présence d'un tiers peut être utile, mais plutôt pour soutenir le candidat que l'employeur. L'association Transfer a ainsi testé les résultats favorables, pour les personnes les plus en difficulté, de la présence lors de l'entretien d'un chargé de mission de l'association. Il complète et renforce l'argumentaire $\mathrm{du}$ candidat, repositionne l'employeur lorsque ce dernier surestime manifestement les compétences exigées par le poste.

\section{Intégrer les aménagements possibles de l'environnement de travail}

La compétence est dépendante de l'environnement. On peut faire varier l'environnement dans le sens d'une diversification du recrutement. Le recruteur, qui sélectionne, doit passer la main à l'ergonome, qui allège la charge de compétence (de Montmollin, 1972).

Par exemple, en matière de handicap, la réglementation prévoit que l'employeur procède à des aménagements raisonnables des postes de travail. De la même façon, on peut envisager des «aménagements» raisonnables de l'environnement pour les jeunes des quartiers, les non-diplômés, ceux qui parlent mal l'anglais, les personnes sans expérience, les chômeurs de longue durée, etc. L'existence de possibilités de formations dans le cours de l'emploi pourrait alléger de façon importante les exigences de niveau de formation au moment du recrutement.

L'équité des recrutements ne se joue donc pas seulement au moment du recrutement : elle est très dépendante des formes d'organisation du travail dans l'entreprise.

\section{Pluraliser les valeurs}

Si l'entreprise s'efforce, pour des raisons bien compréhensibles, d'unifier les valeurs, de créer une 
culture commune qui facilite la communication et la convergence vers un objectif commun, elle exclut de facto les candidats qui n'ont pas le bon format.

Pluraliser les valeurs, outre le fait de faciliter la diversité des personnes, présente deux avantages pour l'entreprise :

- Permettre de gérer plus souplement l'incertitude sur les valeurs qui valent vraiment ;

- Favoriser un meilleur engagement des salariés, moins contraints par un format imposé de valeurs.

Il faut pluraliser les méthodes de recrutement pour réviser les conventions (Ghirardello, van der Plancke, 2006). Cela passe en particulier par une pluralisation des canaux de recrutement. L'ouverture à un nouveau canal de recrutement fait émerger de nouvelles valeurs : d'une part, chaque canal incorpore certaines valeurs; d'autre part, le fait même de rompre avec des routines bien ancrées induit de la critique et de la réflexivité. Par exemple, une entreprise qui rompt avec son habitude de recruter des ingénieurs de telle école pour prospecter des étudiants de l'Université s'ouvre ainsi à de nouvelles valeurs, chacune de ces filières portant des valeurs différentes; et, plus largement, entame un processus d'expérimentation, de recherche de nouvelles solutions, de critique des méthodes.

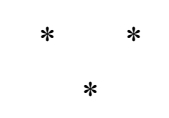

Dans cet article, nous avons prospecté la complexité des opérations d'évaluation réalisées lors des recrutements. L'accent est en général mis sur la dimension cognitive de l'évaluation, bien mesurer certaines caractéristiques des candidats. Il faut aussi voir une dimension que l'on peut qualifier de sociale: les principes d'évaluation sont référés à des situations de coordination, qui demandent à être interprétées. Enfin, l'évaluateur doit non seulement faire preuve de justesse (dimension cognitive), mais aussi de justice (dimension éthique). Il en résulte une triple incertitude : cognitive (il faut inférer la compétence à partir d'une information fragmentaire), sociale (il faut bien identifier la situation de coordination dans laquelle le candidat sera inscrit), éthique (il faut se débrouiller dans le problème compliqué de la justice d'une procédure d'évaluation). La notion de convention de qualité a été mobilisée pour prospecter toute la complexité de ces opérations. La pluralité des situations sociales de coordination et des principes de justice induit la pluralité des conventions de qualité.

La mesure des compétences lors d'un recrutement est ainsi conditionnée par un choix réfléchi entre conventions de qualité. Elle engage des valeurs, donc une forme d'arbitrage entre conventions qui échappe au calcul. Les conventions incluent également des principes de justice : lutte contre la discrimination et plus largement contre les formes d'évaluation inéquitables. On est loin, comme on le voit, de la mesure d'une grandeur « dans la nature », tels une taille ou un poids. De ce fait, les sciences sociales ont beaucoup à apporter à ce sujet important, il s'agit d'évaluer les personnes (Gould, 1983), et peu traité par ces disciplines.

Nous avons exploré, sur un plan normatif, les modes de recrutement qui satisfont ces contraintes de justesse et de justice des évaluations.

Pourquoi les employeurs se plieraient-ils à de telles contraintes? N'y a-t-il pas abondance de candidats, voire surabondance? Cette situation risque d'être un piège. Elle peut renforcer les comportements illégitimes à l'égard des candidats, ce qui a nécessairement des conséquences à terme sur le contrat social. Outre le développement de méthodes illégitimes de la part des candidats que ces comportements des employeurs favorise (CV «bidonnés », multiplication non justifiée des candidatures, etc.), on peut penser que les conditions d'embauche ont une influence profonde sur la façon dont un salarié s'engage dans l'entreprise. On sait bien, par ailleurs, que l'existence d'un niveau de chômage important n'exclut pas des pénuries de main-d'œuvre. On peut penser que la croissance est aujourd'hui limitée par l'insuffisance du travail, et que ces tensions s'aggraveront à l'avenir. Notre hypothèse est que la sursélection est source de pénurie et que des comportements de recrutement plus ouverts et diversifiés réduiraient ces tensions.

De plus, ces comportements auraient des conséquences importantes sur le chômage. Un recrutement plus diversifié induit une répartition plus équitable du chômage entre les différentes catégories de 
personnes, au lieu de le polariser sur une fraction de la population, suivant des processus qui débouchent sur le chômage de longue durée, problème social majeur. Il n'est pas exclu qu'il y ait également un effet favorable sur le volume de chômage: un comportement moins sélectif peut rehausser l'antici- pation de productivité d'une partie des personnes et donc étendre l'espace des recrutements rentables. La voie préconisée ne suppose pas un basculement dans le social : les exigences de justesse et de justice relèvent simplement de ce qu'il est raisonnable de faire pour un acteur économique.

\section{Bibliographie}

Akerlof GA. (1970), «The market for "lemons": quality uncertainty and the market mechanism ", Quarterly Journal of Economics, 84, 3, pp. 488-500.

Akerlof G.A. (1982), « Labor contract as partial gift exchange », Quarterly journal of economics, 87, pp. 543-569.

Benetti C., Cartelier J. (1980), Marchands, salariat et capitalistes, Paris, Maspero.

Bessy C., Eymard-Duvernay F. (Éds), (1997), Les intermédiaires $d u$ marché du travail, Cahiers du Centre d'études de l'emploi, PUF, Paris.

Bessy C., Eymard-Duvernay F., De Larquier G., Marchal E. (Éds) (2001), Des marchés du travail équitables? Une approche comparative France Royaume-Uni, Bruxelles, PIE-Peter Lang.

Boltanski L. et Thevenot L. (1991), De la justification. Les économies de la grandeur, Gallimard, Paris.

Boltanski L., Chiapello E. (1999), Le nouvel esprit du capitalisme, Paris, Gallimard.

Boltanski L., Thevenot L. (Éds.) (1989), Justesse et justice dans le travail, Paris, CEE-PUF.

Castra D., Valls F. (2007), L'insertion malgré tout. L'intervention sur l'offre et la demande. 20 ans d'expérience, Toulouse, Octarès.

Chateauraynaud F. (2003), Prospéro. Une technologie littéraire pour les sciences humaines, Paris, CNRS Éditions.
Doeringer P., Piore M. (1971), Internal labour market and manpower analysis, DC Heath Lexinghton.

Eymard-Duvernay F., Marchal E. (1997), Façons de recruter. Le jugement des compétences sur le marché du travail, Paris, Métailié.

Eymard-Duvernay F., Marchal E. (2000), "Qui calcule trop finit par déraisonner: les experts du marché du travail», Sociologie du travail, 42, pp. 411-432.

Eymard-Duvernay F. (2005), «Conventions de qualité du travail et chômage », Économies et sociétés, série Économie du travail, $\mathrm{n}^{\circ} 26$, pp. 1381-1409.

Gautié J., Godechot O., Sorignet P.-E. (2005), « Arrangement institutionnel et fonctionnement du marché du travail. Le cas de la chasse de tête», Sociologie du travail, 47, pp. 383-404.

Ghirardello A., (2003), La discrimination sur le marché du travail : du problème de coordination à l'égalité dans l'emploi, thèse de sciences économiques, université Paris X Nanterre.

Ghirardello A., van der Plancke V. (2006), « Analyse de la discrimination à l'embauche. Pluraliser les actions positives pour réviser les conventions ", in L'économie des conventions, méthodes et résultats (sous la dir. de F. Eymard-Duvernay), Tome 2, Paris, La Découverte, pp. 145-158.

Gould S.J. (1983), La mal-mesure de l'homme, Paris, Ramsay. 
Levy-Leboyer C. (1990), «L'évaluation du personnel: développements récents et orientations nouvelles », Revue française de gestion, 79, pp. 30-36.

Lyon-Caen G. (1992), Les libertés publiques et l'emploi, Paris, La Documentation française.

Marchal E., Mellet K., Rieucau G. (2007), « Job board toolkits : Internet matchmaking and changes in job advertisements », Human Relations, vol. 60, $\mathrm{n}^{\circ}$ 7, pp. 1091-1113.

Marchal E., Rieucau G. (2006), «Les a priori de la sélection professionnelle : une approche comparative», in L'économie des conventions, méthodes et résultats (sous la dir. de F. Eymard-Duvernay), Tome 2, Paris, La Découverte.

Marchal E., Torny D. (2003), «Des petites aux grandes annonces : Évolution du marché des offres d'emploi (1960-2000)», Travail et Emploi, n 95, pp. 59-72.

Maurice M., Sellier F., Silvestre J.-J. (1982), Politique d'éducation et organisation industrielle en
France et en Allemagne : essai d'analyse sociétale, PUF.

Mellet K. (2006), Les marchés numériques $d u$ travail, Thèse pour le doctorat en sciences économiques, université Paris X-Nanterre.

De Montmollin M. (1972), Les psychopitres, Paris, PUF.

Orlean A. (1991), « Logique Walrasienne et incertitude qualitative: des travaux d'Akerlof et Stiglitz aux conventions de qualité », Économies et Sociétés, Série Économica, 14, pp. 137-160.

Rawls J. (1987), Théorie de la justice, Paris, Seuil.

Salognon M. (2005), Évaluation de la qualité du travail et chômage de longue durée, Thèse pour le doctorat en sciences économiques, Paris X.

De Schutter O. (2001), Discriminations et marché du travail, Bruxelles, PIE-Peter Lang.

Stiglitz J.E. (1987), « The causes and consequences of the dependence of quality on price », Journal of Economic Literature, 25, pp. 1-48.

\section{Résumé}

\section{Justesse et justice dans les recrutements François Eymard-Duvernay}

La question des recrutements occupe une place modeste dans les approches d'économie ou de sociologie. Pourtant, la sélection opérée lors de recrutements joue un rôle important sur le chômage. Le présent article, adoptant une démarche interdisciplinaire, se focalise sur les opérations d'évaluation et de sélection au cœur des recrutements. Il les analyse dans leurs différentes dimensions : cognitive (il faut mesurer correctement des données), sociale (les conceptions de la compétence sont relatives à des contextes sociaux spécifiques), éthique (l'évaluation de la compétence doit obéir à des principes de justice). La notion de convention de compétence permet de synthétiser ces dimensions de l'évaluation. L'approche fait également état des travaux empiriques récents menés dans ce cadre.

\section{Mots clés}

Recrutement, évaluation, compétence, éthique, économie des conventions

Journal of Economic Literature: M 54 - Labor Management ; J 01 - Labor Economics : General 


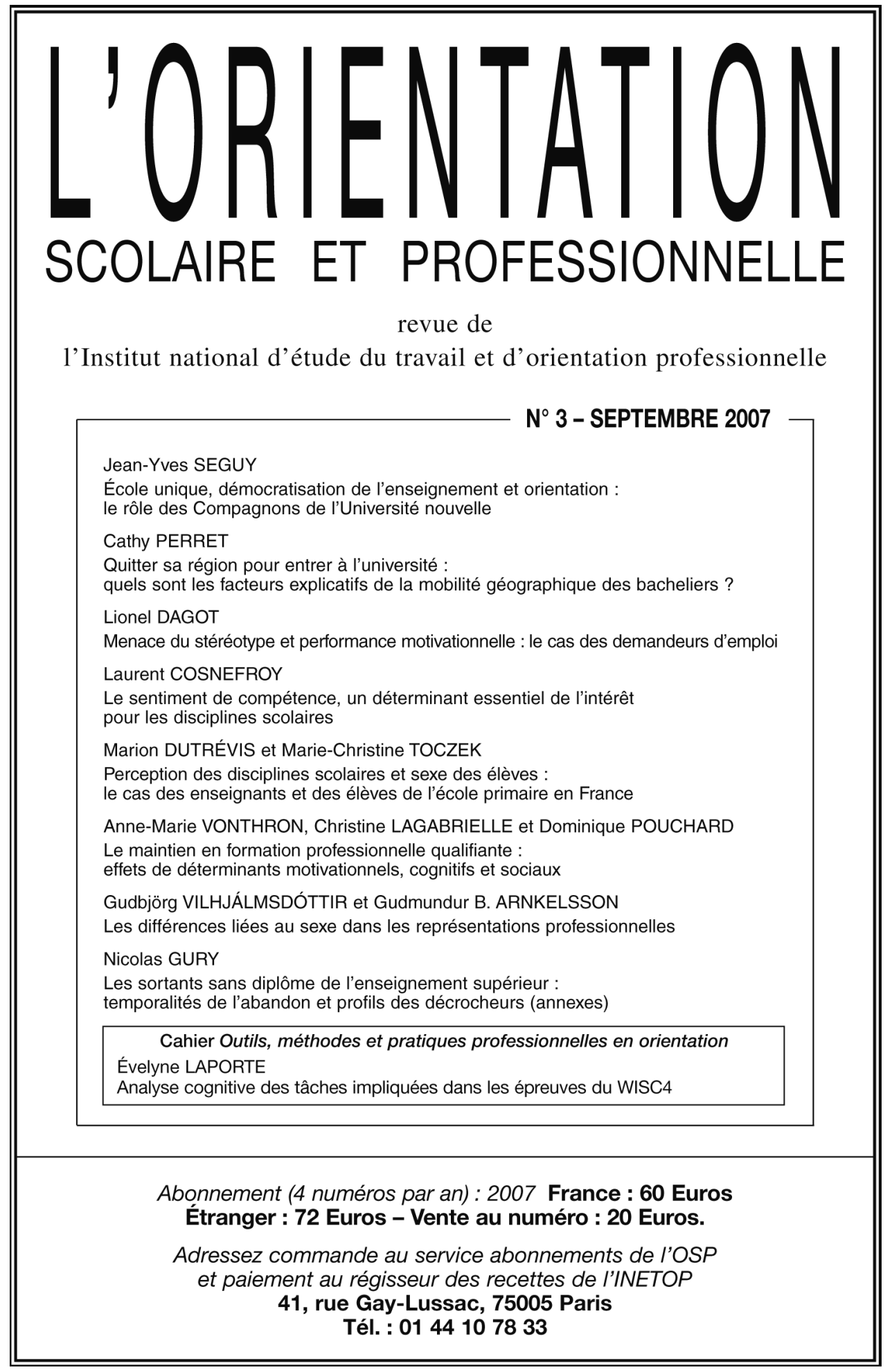

